\title{
Anti-inflammatory effects of Chinese medicinal herbs on cerebral ischemia
}

\author{
Shan-Yu Su ${ }^{1}$ and Ching-Liang Hsieh ${ }^{1,2,3^{*}}$
}

\begin{abstract}
s
Recent studies have demonstrated the importance of anti-inflammation, including cellular immunity, inflammatory mediators, reactive oxygen species, nitric oxide and several transcriptional factors, in the treatment of cerebral ischemia. This article reviews the roles of Chinese medicinal herbs as well as their ingredients in the inflammatory cascade induced by cerebral ischemia. Chinese medicinal herbs exert neuroprotective effects on cerebral ischemia. The effects include inhibiting the activation of microglia, decreasing levels of adhesion molecules such as intracellular adhesion molecule-1, attenuating expression of pro-inflammatory cytokines such as interleukin-1 $\beta$ and tumor necrosis factor- $\alpha$, reducing inducible nitric oxide synthase and reactive oxygen species, and regulating transcription factors such as nuclear factor- $\kappa$ B.
\end{abstract}

\section{Introduction}

Activation of multiple inflammatory cascades accounts for the progressing of ischemia stroke [1]. After cerebral ischemia, energy depletion and necrotic neuron death in the local ischemic area start the inflammatory cascades. The reperfusion generates reactive oxygen species (ROS) that induce the production of cytokines and chemokines leading peripheral leukocytes to influx into the cerebral parenchyma and activate endogenous microglia. Then cellular immunity, adhesion molecules, inflammatory mediators, transcriptional factors participate in the inflammatory process.

Anti-inflammatory treatment that inhibits specific steps of the inflammatory cascade is a new strategy for improving outcome after ischemia stroke [2-4]. The anti-inflammatory agents, including a variety of natural products used in Chinese medicine, have been shown to be able to prevent or treat ischemic stroke, by decreasing the infarct area and neurological deficiency [5]. These natural products are documented as anti-oxidative, anti-inflammatory, anti-apoptotic and neuro-functional regulatory agents [5]. Some active ingredients isolated from these herbs have been identified and demonstrated to have neuroprotective actions. Some of these compounds are andrographolide

\footnotetext{
* Correspondence: clhsieh@mail.cmuh.org.tw

'Department of Chinese Medicine, China Medical University Hospital,

Taichung 40447, Taiwan

Full list of author information is available at the end of the article
}

isolated from Andrographis paniculata (Chuan-xinlian), oxymatrine isolated from Sophora flavescens (Ku-shen), quercetin isolated from Sophora japonica (Huai-hua), ferulic acid isolated from both Angelica sinensis (Dang-gui) and Ligusticum wallichii (Chuanxiong), tetramethylpyrazine isolated from Ligusticum wallichii (Chuan-xiong), paeonol and paeoniflorin isolated from Paeonia lactiflora (Bai-shao), shikonin isolated from Lithospermum erythrorhizon (Zi-cao), vanillin, 4-hydroxybenzyl alcohol and 4-hydroxybenzyl aldehyde isolated from Gastrodia elata (Tain-ma), puerarin from Radix Puerariae (Pueraria lobata; Gegen), polydatin and emodin-8-O- $\beta$-D-glucoside isolated from Polygonum cuspidatum (Hu-zhang), tanshinone IIA isolated from Salviae miltiorrhiza (Dan-shen), wogonin isolated from Scutellaria baicalensis (Huangqin) and apocynin isolated from Picrorhiza kurroa (Hu-huang-lian) (Figure 1).

This article reviews the current roles of Chinese medicinal herbs as well as their ingredients in the inflammatory cascade induced by cerebral ischemia. Using cerebral ischemia (OR ischemic stroke) AND herb (OR traditional Chinese medicine) AND inflammation (OR inflammatory OR immunity) as the keywords, we search the English databases including PudMed, Medline, and Cochrane library from 1980 to 2010, generating 77 articles from the initial search. 


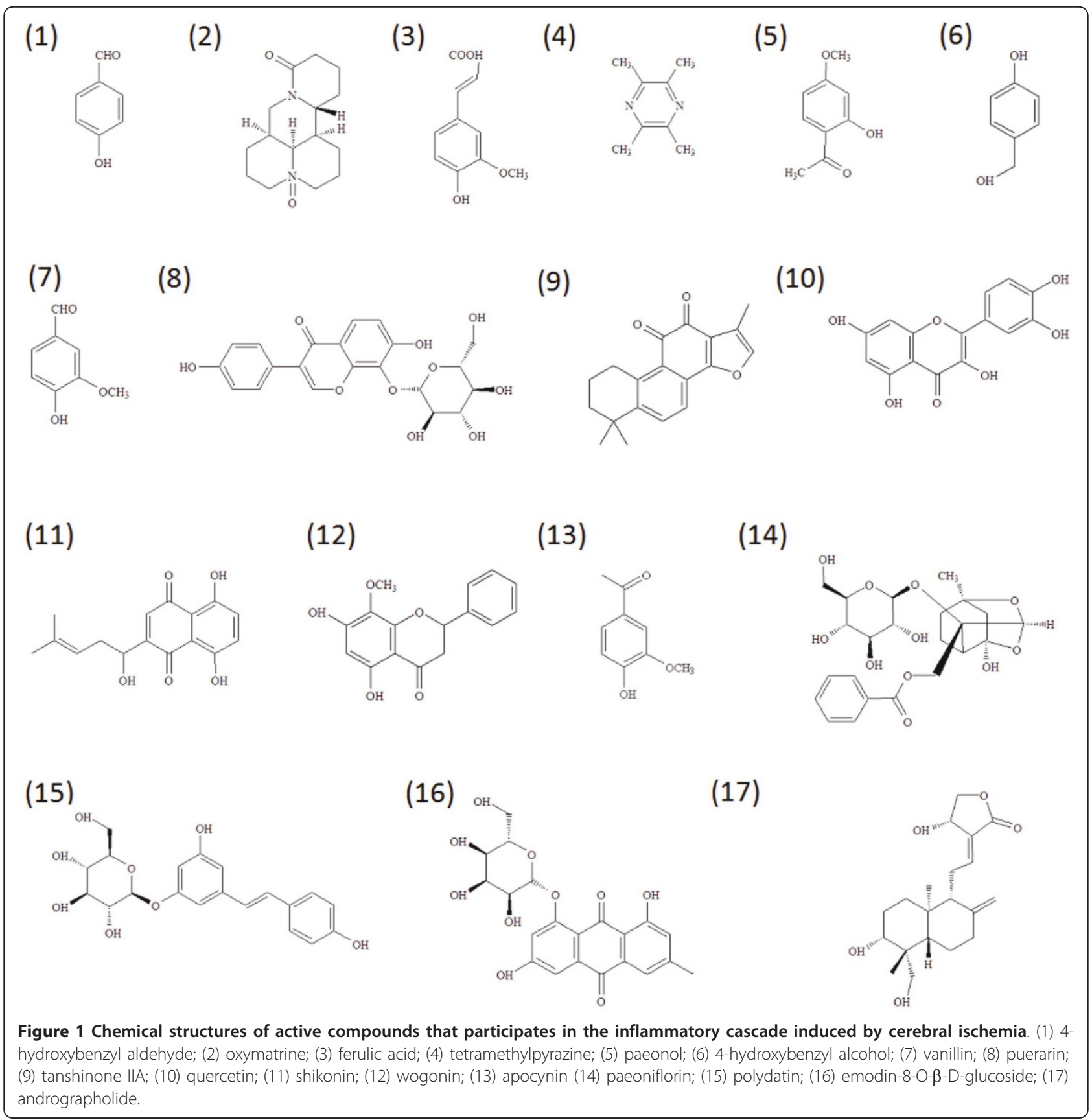

\section{Chinese medicinal herbs for reducing inflammation in cerebral ischemia} Inhibition of cellular immunity

After the onset of ischemia, cellular immunity, including that executed by blood-derived leukocytes, microglia and astrocytes are activated. Immune cells accumulate in the brain tissues, leading to neuronal injury. Leukocytes are the first inflammatory cells recruited into ischemic brain tissues and potentiate injury by secreting deleterious substances and inflammatory mediators [6]. Microglia are activated after ischemia and undergo morphological transformation into phagocytes followed by stimulation of toll-like receptors 4 (TLR-4) [7].

Andrographolide, a diterpenoid lactone isolated from Andrographis paniculata that is traditionally used to treat fever [8], reduces the activation of microglia in a cell model of primary rat mesencephalic neuron-glia culture [9]. Apocynin, the main active ingredient of Picrorhiza kurroa, blocks microglia activation in a chemical ischemic model of cultured neuroblastoma cells [10] (Table 1). In a rat model of permanent middle cerebral artery occlusion (pMCAo), andrographolide reduces 
Table 1 Medicinal herbs that suppress cellular responses induced by cerebral ischemia

\begin{tabular}{ll}
\hline \hline $\begin{array}{l}\text { Targeted cells/ } \\
\text { molecules }\end{array}$ & Herb or compound \\
\hline $\begin{array}{l}\text { Microglia/ } \\
\text { microphage }\end{array}$ & $\begin{array}{l}\text { andrographolide [30], paeonol [11], wogonin [34], } \\
\text { Sophora japonica [13], Angelica sinensis [51], Panax } \\
\text { Notoginseng [15], apocynin [10] }\end{array}$ \\
Astrocytes & Zizyphus jujube [16] \\
$\begin{array}{l}\text { Adhesion } \\
\text { molecules } \\
\text { Selectins } \\
\text { Integrins }\end{array}$ & $\begin{array}{l}\text { polydatin [24] } \\
\text { ICAM-1 }\end{array}$ \\
& $\begin{array}{l}\text { ferulic acid [26], polydatin [24], Panax Notoginseng } \\
\text { saponins [22], apocynin [20], paeoniflorin [23] }\end{array}$ \\
\hline
\end{tabular}

the infarct area at $0.1 \mathrm{mg} / \mathrm{kg}$ by reducing the activation of microglia; meanwhile, the inflammation process induced by pMCAo is also suppressed. Paeonol, the active ingredient of Paeonia lactiflora traditionally used to treat inflammation-associated allergic rhinitis, otitis and appendicitis, reduces the infarct area and improves the neurological outcome in a transient middle cerebral artery occlusion (tMCAo) rat model by inhibiting the activation of microglia [11]. The aqueous crude extracts of Sophora japonica, Panax notoginseng and Zizyphus jujuba reduce the infarct area in a tMCAo model by modulating cellular immunity. Sophora japonica, an anti-oxidative, anti-inflammatory, anti-platelet aggregation and cardiovascular protective agent [12], reduces activated microglia cells labeled by ED1 [13]. Panax notoginseng, which is beneficial to the cardiovascular system and is used routinely to treat acute ischemia stroke in China [14] decreases microglical density in the peri-infarct region [15]. Zizyphus jujuba protects ischemic damage by decreasing the gliosis of astrocytes and microglia in the CA1 region four days after ische$\mathrm{mia} /$ reperfusion [16].

\section{Inhibition of adhesion molecules}

Adhesion molecules are crucial in the recruiting of leukocytes into the brain parenchyma after ischemia. The interaction between leukocytes and the vascular endothelium is mediated by three main groups of cell adhesion molecules, namely selectin (P-selectin, E-selectin, and L-selectin), the immunoglobulin superfamily including intra-cellular adhesion molecule-1 (ICAM-1), ICAM-2 and vascular cell adhesion molecules-1 (VCAM-1), and integrins [17]. The suppression of adhesion molecules is considered an important therapeutic target [18].

Apocynin, isolated from Picrorhiza kurroa, attenuates both cerebral infarct volume and neurological defect in ischemia/reperfusion rat models [10,19-21]. The neuroprotection by apocynin is accompanied by the suppression of ICAM in ischemic regions [20]. Treatment of saponins extracted from Panax notoginseng and paeoniflorin from Paeonia lactiflora inhibits expression of ICAM-1 and MPO activity in a tMCAo rat model [22,23]. Polydatin. i.e. 3,4',5-trihydroxystilbene-3- $\beta$ mono-D-glucoside, one of the components isolated from Polygonum cuspidatum, protects the brain from leukocyte recruitment after ischemia injury by decreasing adhesion molecules, including ICAM-1, VCAM-1, Eselectin, L-selectin and integrins [24]. Polygonum cuspidatum is traditionally used in inflammatory diseases, including dermatitis, abscess and hepatitis [25]. Ferulic acid, the active compound of Angelica sinensis and Ligusticum wallichii, exhibits similar effects. Intravenous injection of ferulic acid ( 80 and $100 \mathrm{mg} / \mathrm{kg}$ ) at the beginning of tMCAo reduces cerebral infarct area and improves neurological functions measured by neurological deficit scores in rats by blocking ICAM-1 activity [26].

\section{Regulation of cytokines}

Pro-inflammatory cytokines drive the inflammatory process and aggravate inflammation. Cytokines that participate in the inflammation after cerebral ischemia include

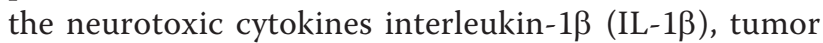
necrosis factor-alpha (TNF- $\alpha$ ), neuroprotective cytokines interleukin-6 (IL-6), interleukin-10 (IL-10) and transforming growth factor- $\beta$ [27]. Among these cytokines, IL- 1 and TNF- $\alpha$ are shown to be decreased by several herbs (Table 2). Total saponins extracted from Panax notoginseng reduce IL-1 activity [28]. Paeonol, apocinin and the aqueous extract of Sophora japonica reduce IL$1 \beta$ immune-reactive cells in brain parenchyma of a tMCAo model $[13,20,29]$. Andrographolide, paeoniflorin and andrographolide inhibit both TNF- $\alpha$ and IL- $1 \beta$ simultaneously $[23,30]$. Both puerarin, the principal bioactive isoflavonoid derived from peuraria lobata and wogonin (5,7-dihydroxy-8-methoxyflavone) isolated from the root of Scutellaria baicalensis exert neuroprotection by inhibiting TNF- $\alpha$. Radix puerariae is a medicinal plant used as antipyretic, antidiarrhetic, diaphoretic and antiemetic agents [31]. Ethanol extract of Radix puerariae acts as an anti-depressant in mice undergoing cerebral ischemia/reperfusion [31]. Puerarin reduces infarct volume in the tMCAo rat model at 50 $\mathrm{mg} / \mathrm{kg}$. The associated mechanisms include the ability to down-regulate TNF- $\alpha$ [32]. Methanol extracts from the dried roots of Scutellaria baicalensis $(0.1-10 \mathrm{mg} / \mathrm{kg}$ ) significantly protect $\mathrm{CA} 1$ neuronal cells against transient forebrain ischemia [33]. Wogonin induces TNF- $\alpha$ and protects hippocampal neuron from death in a transient global ischemia by four-vessel occlusion in rats [34]. 
Table 2 Herbs or herbal compounds that suppress the production of inflammatory mediators and transcription factors activated by cerebral ischemia

\begin{tabular}{|c|c|}
\hline $\begin{array}{l}\text { Targeted } \\
\text { molecules }\end{array}$ & Herb or compound \\
\hline \multicolumn{2}{|l|}{$\begin{array}{l}\text { Inflammatory } \\
\text { meciators }\end{array}$} \\
\hline \multicolumn{2}{|l|}{ Cytokines } \\
\hline $\mathrm{IL}-1 \beta$ & $\begin{array}{l}\text { andrographolide [30], paeonol [11],Sophora japonica } \\
{[13], \text { Panax Notoginseng saponins [28], apocynin [20], }} \\
\text { Scutellaria baicalensis flavonoid [54] }\end{array}$ \\
\hline TNF- $\alpha$ & andrographolide [30], puerarin [32], wogonin [34] \\
\hline \multicolumn{2}{|l|}{ NO } \\
\hline iNOS & $\begin{array}{l}\text { ferulic acid [38], puerarin [32], tetramethylpyrazine } \\
\text { [40], wogonin [34], Panax Notoginseng [15] }\end{array}$ \\
\hline ROS & $\begin{array}{l}\text { Salviae Miltiorrhiza[41], 4-hydroxybenzyl alcohol [48], } \\
\text { Angelica sinensis [51], tanshinone IIA [45] }\end{array}$ \\
\hline MPO & $\begin{array}{l}\text { ferulic acid [26], tetramethylpyrazine [52], } \\
\text { Anemarrhena asphodeloides [53], Panax Notoginseng } \\
\text { saponins [22] }\end{array}$ \\
\hline SOD & $\begin{array}{l}\text { shikonin [57], paeonol [29], emodin-8-O- } \beta \text {-D- } \\
\text { glucoside [58], Zizyphus jujube [16], Scutellaria } \\
\text { baicalensis flavonoid [54] }\end{array}$ \\
\hline CAT & Scutellaria baicalensis isoflavoid [54], shikonin [57] \\
\hline GSH & shikonin [57] \\
\hline $\mathrm{OH} \cdot$ & tetramethylpyrazine [40] \\
\hline NADPH & Apocinin [20] \\
\hline MMP & quercetin [62], Panax Notoginseng saponins [64] \\
\hline \multicolumn{2}{|l|}{$\begin{array}{l}\text { Transcription } \\
\text { factors }\end{array}$} \\
\hline $\mathrm{NF}-\kappa \mathrm{B}$ & $\begin{array}{l}\text { andrographolide [30], oxymatrine [81], feulic acid } \\
\text { [26], paeoniflorin [82], wogonin [80],Panax } \\
\text { Notoginseng [15], apocynin [20] }\end{array}$ \\
\hline P38 & Oxymatrine [73], ferulic acid [38] \\
\hline
\end{tabular}

\section{Inhibition of oxidative stress and NO}

After cerebral ischemia, reperfusion leads to the generation of ROS by several enzymes. Superoxide anion is generated by cyclooxygenase (COX), xanthine dehydrogenase, xanthine oxidase, nicotinamide adenine dinucleotide phosphate (NADPH) oxidase and hypochlorous; hydrogen peroxide $\left(\mathrm{H}_{2} \mathrm{O}_{2}\right)$ are generated by myeloperoxidase (MPO) and monoamine oxidase (MAO). Among these, superoxide anion reacts with $\mathrm{NO}$ to generate peroxynitrite [35]. ROS stimulates ischemic cells to secrete inflammatory cytokines and chemokines which cause adhesion molecule up-regulation in the cerebral vasculature and peripheral leukocyte recruitment. Once activated, inflammatory cells release a variety of cytotoxic agents such as cytokines, matrix metalloproteinases (MMPs), NO and ROS [36]. The MMPs are proteases that break down extracellular proteins such as collagen, leading to extracellular matrix remodeling in the inflammatory response [37]. Among the three isoforms of
NOS, namely inducible NOS (iNOS), neuronal NOS (nNOS) and endothelial NOS (eNOS), iNOS expression is restricted to cells involved in inflammatory responses such as circulating leukocytes, microglia, and astrocytes and therefore, iNOS is thought to be the most contributive NOS contributes to the ischemic injury via generating nitric oxide (NO) [36].

Herbs and their ingredients that exert neuroprotective effects via inhibiting NO include ferulic acid, puerarin, tetramethylpyrazine, wogonin and Panax notoginseng. Intravenous injection of ferulic acid ( 80 and $100 \mathrm{mg} / \mathrm{kg}$ ) at the beginning of tMCAo abrogates the elevation of nNOS, iNOS and p38 activation, leading to the decrease of the number of relevant apoptotic cells in the ischemia brain [38]. The inhibition of TNF- $\alpha$ by puerarin is followed by the inhibition of iNOS expression and active caspase- 3 formation, resulting in a reduction in the infarct volume in ischemia-reperfusion brain injury [32]. Wogonin reduces iNOS after cerebral ischemia [34]. Tetramethylpyrazine, which is isolated from Ligusticum wallichiit, protects brain from ischemia insult [39]via decreasing nitrotyrosine, iNOS and hydroxyl radical formation [40].

In a tMCAo rat model, the luminal luciferase count in the brain parenchyma is suppressed by Salviae miltiorrhiza [41], which is used as a common herb to treat acute ischemic stroke [42]. Aqueous extract of Salviae miltiorrhiza reduces the infarct area and preserves pyramidal cells in tMCAo rats [43] as well as the NOS gene expression in the cerebral cortex and caudate-putamen in the ischemic brain [44]. The active component of Salviae miltiorrhiza, tanshinone IIA (10 mg/kg, i.p.), exhibits high anti-oxidative activities in a rat model of hypoxia-ischemia encephalopathy, in which the rat is exposed to a low oxygen environment $(8 \%)$ and the right common carotid artery is ligated [45]. The same neuronal protective effect exists in the neonatal brain with hypoxia-ischemia injury [45].

Aqueous extract of Gastrodia elata, which is widely used to treat convulsive disorders, protects the brain from ischemia in rat and in gerbil models $[46,47]$. The active compound isolated from Gastrodia elata, 4hydroxybenzyl alcohol, may explain the neuro-protection activity. It increases the antioxidant protein including protein disulfide isomerase and 1-Cys peroxiredoxin (1-Cys Prx) [46]. The down-regulation of 8-hydroxy-2'deoxyguanosine suggests that 4-hydroxybenzyl alcohol scavenges free radicals [48], which may be related to its inhibition of apoptosis in a rat tMCAo model [49]. Another two compounds isolated from Gastrodia elata, namely vanillin and 4-hydroxybenzyl aldehyde, also show neuroprotective ability in cerebral ischemia. Among the three compounds isolated from Gastrodia elata, vanillin-treated animals have the greatest neuronal 
survival after ischemia insult [48]. Treatment of Angelica sinensis $(5 \mathrm{~g} / \mathrm{kg})$ simultaneously with cerebral ischemia reduces the infarct area caused by tMCAo [50]. Oral feeding of aqueous extracts of Angelica sinensis for seven days $(250 \mathrm{mg} / \mathrm{kg} /$ day $)$ attenuates oxidative stress in the brain [51].

Several Chinese medicinal herbs produce their neuroprotective effects via suppression of MPO, including ferulic acid, tetramethylpyrazine, Anemarrhena asphodeloides and Panax notoginseng saponins. At the beginning of tMCAo, intravenous injection of ferulic acid ( 80 and $100 \mathrm{mg} / \mathrm{kg}$ ) suppresses the expression of MPO [26]. The protective effects of tetramethylpyrazine and Panax notoginseng saponins are associated with the reduced ischemia/reperfusion induced MPO activity levels, indicating that Panax notoginseng saponins decreases the production of ROS and ROS-related inflammatory activity [52]. The aqueous extract of Anemarrhena asphodeloides increases MPO activity and protects animals from ischemia/reperfusion injury with a therapeutic time window from one hour prior to reperfusion to two hours after reperfusion [53].

Chinese medicinal herbs that suppress ROS by increasing the activity of antioxidative enzymes include Scutellaria baicalensis flavonoid, shikonin, paeonol, emodin-8-O- $\beta$-D-glucoside and Zizyphus jujube extract. In a permanent cerebral ischemic model in rats, in which the bilateral common carotid arteries are ligated, oral feeding of total flavonoid $(17.5-70 \mathrm{mg} / \mathrm{kg}$ ) from $\mathrm{Scu}$ tellaria baicalensis increase SOD and catalase (CAT) activity in the hippocampus and cerebral ischemia cortex [54]. Paeonol as well increases superoxide dismutase (SOD) activity after cerebral ischemia [29]. Shikonin is a naphthoquinone pigment isolated from Lithospermum erythrorhizon, which is traditionally used to heal wounds and treat inflammatory dermatological diseases $[55,56]$. Shikonin protects the brain from ischemia in the tMCAo mouse model by acting as an antioxidant. It upregulates SOD, catalase, glutathione peroxidase (GSH$\mathrm{Px}$ ) activities and down-regulates glutathione (GSH)/glutathione disulfide (GSSG) ratio [57]. Paeonol also exerts anti-oxidative activity by increasing superoxide dismutase (SOD) activity [29]. Emodin-8-O- $\beta$-D-glucoside, extracted from Polygonum cuspidatum, increases the total antioxidant capacity of cells after cerebral ischemia. Increased SOD level and decreased MDA level reduce infarct area and neurological defect [58]. The antiinflammatory effects of Zizyphus jujuba come from the reduction of hydroxynonenal level, an indicator of lipid peroxidation and elevation the SOD level [16]. Several compounds are isolated from Zizyphus jujuba, such as jujuboside [59], triterpenic acid [60] and saponins [61], but the specific active compound responsible for the neuroprotective effects has yet to be identified. As an
NADPH oxidase inhibitor, apocinin exerts neuroprotective effects by the blockage of ROS production in leukocytes via the inhibition of NADPH oxidase, leading to the elimination of cytokine and adhesion molecule production [20].

Some Chinese medicinal herbs have effects on MMP9. Quercetin, one of the flovonoids isolated from Sophora japonica, protects the blood-brain barrier and elevates MMP-9 levels in the photothrombotic animal model while the level of MMP-2 is not regulated by quercetin [62]. Total saponins extracted from Panax notoginseng reduces the expression of caspase- 1 and caspae-3, resulting in the attenuation of apoptosis [63]. Panax notoginseng saponins reduce protein levels of MMP-9 in a mouse tMCAo model [64]. Three major bioactive saponins have been identified to be ginsenoside $R g_{1}$, ginsenoside $R b_{1}$ and notoginsenoside $R_{1}$ [65].

\section{Regulation of chemokines}

Chemokines have a deleterious role by increasing leukocyte infiltration after stroke [66]. Arachidonic acid (AA) cascade is a downstream signal pathway of immune cells initiated via phospholipase A2 (PLA2) and phospholipase $\mathrm{C}$ (PLC) which is activated by calcium accumulation caused by cessation of energy by ischemia [67]. PLA2 hydrolyzes glycerophospholipids to release AA, which is metabolized through two different pathways via cyclooxygenase (COX) or lipoxygenase (LOX). The COX pathway converts AA to prostaglandin $\mathrm{H} 2$ (PGH2) which is then metabolized into eicosanoids, including prostacyclin (PGI2), thromboxane A2 (TXA2), prostaglandin E2 (PGE2) and prostaglandin D2 (PGD2) [68]. These eicosanoids affect vasomotor regulation and increase microvascular and blood-brain barrier (BBB) permeability $[69,70]$. AA is also converted to 5-hydroperoxyeicosateraenoic acid (5-HPETE) by 5-LOX. 5-HPETE is then metabolized to leukotrienes to mediate chemoattraction, brain edema and BBB permeability [71].

Ingredients from Sophora flavescens, Panax notoginseng, Andrographis paniculata, and Ligusticum wallichii block chemokines after cerebral ischemia. Sophora flavescens is used for anti-oxidation, anti-bacterial, antiinflammation, anti-fever, anti-arrhythmia, anti-asthma, anti-ulcer and anti-neoplasm [72]. One of the major alkaloids of Sophora flavescens, oxymatrine, reduces the overexpression of phosphorylated p38, 12/15 LOX and cytosolic PLA2 [73]. The alkaloid-free fraction extracted by EtOAc containing two major flavonoids kurarinone (45.5\%) and sophoraflavone G (14.7\%) protects the brain from injury of pMCAo [74]. The underlying protective mechanisms of Panax notoginseng comprise the inhibition COX-2 via blocking the nuclear factor $-\kappa \mathrm{B}(\mathrm{NF}-\kappa \mathrm{B})$ pathway [15]. Andrographolide reduces the infarct area in a rat model of pMCAo by decreasing AA metabolism 
into PGE [30]. Moreover, tetramethylpyrazine reduces PGE2 levels induced by ischemia/reperfusion [52] (Figure 2).

\section{Transcription factors}

During the inflammatory process, activation of a specific transcription factor, including NF- $\kappa \mathrm{B}$, mitogen-activated protein kinase (MAPK), activator protein-1 (AP-1) and regulation of specific gene expression are needed. Many inflammatory genes contain NF- $\kappa \mathrm{B}$ binding site, such as TNF- $\alpha$, ICAM-1, iNOS and IL-6 [75].

Three MAPKs are documented during cerebral ischemia, namely the stress-activated protein kinases/c-Jun $\mathrm{N}$-terminal kinase (SAPK/JNK), p38 MAPK and the extracellular signal-regulated kinases (ERKs). P38 MAPK stabilizes and enhances the translation of mRNA encoding pro-inflammatory protein [76]. The reduction of ERKs is necessary for the recovery from ischemic stroke [77]. Mediated through JNK cascade, AP-1 is activated by the up-regulation of c-fos 30 minutes after the onset of a stroke [78]. The p38 MAP kinase participates in the
mRNA expression of c-jun and c-fos after cerebral ischemia [79].

Several Chinese medicinal herbs block inflammation by inhibiting the NF- $\kappa \mathrm{B}$ pathway, including andrographolide, oxymatrine, feulic acid, paeoniflorin, wogonin, Panax notoginseng and apocynin $[30,80,81]$. Panax notoginseng inhibits inflammatory mediators, including iNOS and COX-2 by blocking the NF- $\kappa$ B pathway [15]. In a chronic cerebral ischemia rat model, in which bilateral carotid arteries are permanently occluded, paeoniflorin $(25 \mathrm{mg} / \mathrm{kg})$ decreases the expression of NF- $\kappa \mathrm{B}$ in astrocyte and microglia within hippocampal area [82]. The protective effect provided by wogonin has been demonstrated in a pMCAo model, in which wogonin reduces the total volume of infarction and improves behavior functions [83], associated with the reduction of NF- $\kappa \mathrm{B}$ activity, but not with the regulation of mitogen-activated protein kinases family members, p38, ERK and JNK [80]. The inhibition of COX-2 by Panax notoginseng may be achieved via blocking the NF- $\kappa \mathrm{B}$ pathway [15]. Apocynin reduces inflammation also via the inhibition



Figure 2 Molecular targets of herbal medicines for interrupting arachidonic acid metabolism. 
of NF- $\kappa \mathrm{B}$ [20]. The reduction of LOX, PLA2 and TLR by oxymatrin may be related to the inhibiting of the NF- $\kappa \mathrm{B}$ and p38 activation [73]. The decreases of ICAM1 and MPO by ferulic acid also considered a result of the suppression of NF- $\kappa \mathrm{B}$ [26] and the inhibition of p38 may lead to the decrease of relevant apoptosis [38].

\section{Clinical trials}

Most clinical trials of Chinese medicine on ischemic stroke test the efficacy of multi-herb formulae. For example, Danqi Piantang Jiaonang containing Salviae miltiorrhiza, Ligusticum wallichii, Angelica sinensis improved neurological recovery in patients after a stroke [84]. A multi-center randomized controlled trial (RCT) suggested Danqi Piantang Jiaonang to increase the scores evaluated by diagnostic therapeutic effects of Apoplexy scoring system in post-stroke rehabilitation and in the recovery of patients with posterior circulation infarction and severe ischemic stroke $[85,86]$. Two other clinical studies for two Chinese herbal formulae, namely Dengzhan Shengmai capsule and Huatuo Zaizao Wan are currently in progress. On the other hand, few single herbs have been tested in clinical trials. In a multi-center, double-blinded, randomized controlled clinical trial of 140 patients suffering subacute ischemic stroke, Panax notoginseng ameliorated neurological deficit and activities of daily living [87]. Chen et al reported that by reviewing several papers including 660 patients in RCTs, Panax notoginseng was safe and beneficial [14]. Salviae miltiorrhiza has been studied in clinical trials; however, the results were inconclusive. A systematic review of 33 Salviae miltiorrhiza trials for acute ischemic stroke did not support the efficacy of Salviae miltiorrhiza in disability improvement after acute ischemic stroke [42]. These clinical trials share similar problems, e.g. lack of placebo-controlled trial and small sample size $[14,42]$.

\section{Conclusions}

Many Chinese medicinal herbs that act on the inflammation process were used to treat ischemia stroke. These herbs suppress inflammatory cascades in cellular immunity, adhesion molecules, cytokines, arachidonic acid, metabolites, NO, ROS, and transcriptional factors. In the future, more clinical trials should be down to Chinese herbs that have been demonstrated effective in animal studies but not been proven in human.

\section{Abbreviations}

AA: arachidonic acid; AP-1: activator protein-1; BBB: blood-brain barrier; COX: cyclooxygenase; 1-Cys Prx: 1-Cys peroxiredoxin; ERKs: extracellular signalregulated kinase; GSH: glutathione; GSH-Px: glutathione peroxidase; GSSG: glutathione disulfide; 5-HPETE: 5-hydroperoxyeicosateraenoic acid; ICAM-1: intra-cellular adhesion molecule-1; ICAM-2: intra-cellular adhesion molecule-2; IL-1: interleukin-1; IL-6: interleukin-6; IL-10: interleukin-10; JNK: c-Jun Nterminal kinase; LOX: lipoxygenase; MAO: monoamine oxidase; MAPK: mitogen-activated protein kinase; pMCAo: permanent middle cerebral artery occlusion; tMCAo: transient middle cerebral artery occlusion; MPO: myeloperoxidase; MMPs: metalloproteinases; NF-kB: nuclear factor-kB; NADPH: nicotinamide adenine dinucleotide phosphate; NO: nitric oxide; eNOS: endothelial nitric oxide synthase; iNOS: inducible NOS; nNOS: neuronal nitric oxide synthase; PG12: prostacyclin; PLA2: phospholipase A2; PGD2: prostaglandin D2; PGE2: prostaglandin E2; PLC: phospholipase C; ROS: reactive oxygen species; SAPK: stress-activated protein kinases; SOD: superoxide dismutase; TLR-4: toll-like receptors 4; TNF-a: tumor necrosis factor-a; TXA2: thromboxane A2; VCAM-1: vascular cell adhesion molecules-1.

\section{Acknowledgements}

This study was supported in part by Taiwan Department of Health Clinical Trial and Research Center of Excellence (DOH100-TD-B-111-004).

\section{Author details}

'Department of Chinese Medicine, China Medical University Hospital, Taichung 40447, Taiwan. ${ }^{2}$ Graduate Institute of Acupuncture Science, China Medical University, Taichung 40402, Taiwan. ${ }^{3}$ Acupuncture Research Center, China Medical University, Taichung, 40402, Taiwan.

\section{Authors' contributions}

CLH designed the study and revised the manuscript. SYS conducted the literature search and drafted the manuscript. Both authors read and approved the final version of the manuscript.

\section{Competing interests}

The authors declare that they have no competing interests.

Received: 19 February 2011 Accepted: 9 July 2011

Published: 9 July 2011

\section{References}

1. Ahmad M, Graham SH: Inflammation after stroke: mechanisms and therapeutic approaches. Trans/ Stroke Res 2010, 1(2):74-84.

2. Morancho A, Rosell A, Garcia-Bonilla L, Montaner J: Metalloproteinase and stroke infarct size: role for anti-inflammatory treatment? Ann N Y Acad Sci 2010, 1207:123-133

3. Stoll G, Kleinschnitz C, Nieswandt B: Combating innate inflammation: a new paradigm for acute treatment of stroke? Ann N Y Acad Sci 2010, 1207:149-154.

4. del Zoppo GJ: Acute anti-inflammatory approaches to ischemic stroke. Ann N Y Acad Sci 2010, 1207:143-148.

5. Wu PF, Zhang Z, Wang F, Chen JG: Natural compounds from traditional medicinal herbs in the treatment of cerebral ischemia/reperfusion injury. Acta Pharmacol Sin 2010, 31(12):1523-1531.

6. Hallenbeck JM: Significance of the inflammatory response in brain ischemia. Acta Neurochir Supp/ 1996, 66:27-31.

7. Downes CE, Crack PJ: Neural injury following stroke: are Toll-like receptors the link between the immune system and the CNS? $\mathrm{Br} J$ Pharmacol 2010, 160(8):1872-1888.

8. Kunwar RM, Shrestha KP, Bussmann RW: Traditional herbal medicine in Far-west Nepal: a pharmacological appraisal. J Ethnobiol Ethnomed 2010, 6(1):35.

9. Wang T, Liu B, Zhang W, Wilson B, Hong JS: Andrographolide reduces inflammation-mediated dopaminergic neurodegeneration in mesencephalic neuron-glia cultures by inhibiting microglial activation. J Pharmacol Exp Ther 2004, 308(3):975-983.

10. Hur J, Lee P, Kim MJ, Kim Y, Cho YW: Ischemia-activated microglia induces neuronal injury via activation of gp91phox NADPH oxidase. Biochem Biophys Res Commun 2010, 391(3):1526-1530.

11. Hsieh CL, Cheng CY, Tsai TH, Lin IH, Liu CH, Chiang SY, Lin JG, Lao CJ, Tang NY: Paeonol reduced cerebral infarction involving the superoxide anion and microglia activation in ischemia-reperfusion injured rats. $J$ Ethnopharmacol 2006, 106(2):208-215.

12. Chen $\mathrm{HN}$, Hsieh $\mathrm{CL}$ : Effects of Sophora japonica flowers (Huaihua) on cerebral infarction. Chin Med 2010, 5:34.

13. Lao CJ, Lin JG, Kuo JS, Chao PD, Cheng CY, Tang NY, Hsieh CL: Microglia, apoptosis and interleukin-1beta expression in the effect of sophora japonica I. on cerebral infarct induced by ischemia-reperfusion in rats. Am J Chin Med 2005, 33(3):425-438. 
14. Chen X, Zhou M, Li Q, Yang J, Zhang Y, Zhang D, Kong S, Zhou D, He L: Sanchi for acute ischaemic stroke. Cochrane Database Syst Rev 2008, , 4: CD006305.

15. Son HY, Han HS, Jung HW, Park YK: Panax notoginseng attenuates the infarct volume in rat ischemic brain and the inflammatory response of microglia. J Pharmacol Sci 2009, 109(3):368-379.

16. Yoo KY, Li H, Hwang IK, Choi JH, Lee CH, Kwon DY, Ryu SY, Kim YS, Kang IJ, Shin HC, Won MH: Zizyphus attenuates ischemic damage in the gerbil hippocampus via its antioxidant effect. J Med Food 2010, 13(3):557-563.

17. del Zoppo GJ: Relationship of neurovascular elements to neuron injury during ischemia. Cerebrovasc Dis 2009, 27(Suppl 1):65-76.

18. Sughrue ME, Mehra A, Connolly ES Jr, D'Ambrosio AL: Anti-adhesion molecule strategies as potential neuroprotective agents in cerebral ischemia: a critical review of the literature. Inflamm Res 2004, 53(10):497-508.

19. Murotomi K, Takagi N, Takeo S, Tanonaka K: NADPH oxidase-mediated oxidative damage to proteins in the postsynaptic density after transient cerebral ischemia and reperfusion. Mol Cell Neurosci 2011, 46(3):681-688.

20. Genovese T, Mazzon E, Paterniti I, Esposito E, Bramanti P, Cuzzocrea S: Modulation of NADPH oxidase activation in cerebral ischemia/ reperfusion injury in rats. Brain Res 2011, 1372:92-102.

21. Tang LL, Ye K, Yang XF, Zheng JS: Apocynin attenuates cerebral infarction after transient focal ischaemia in rats. J Int Med Res 2007, 35(4):517-522.

22. He W, Zhu Z: Effect of Panax notoginseng saponins on intercellular adhesion molecule- 1 expression and neutrophil infiltration in cerebral infarction tissue of rats. Zhong Yao Cai 2005, 28(5):403-405.

23. Tang NY, Liu CH, Hsieh CT, Hsieh CL: The anti-inflammatory effect of paeoniflorin on cerebral infarction induced by ischemia-reperfusion injury in Sprague-Dawley rats. Am J Chin Med 2010, 38(1):51-64.

24. Cheng Y, Zhang HT, Sun L, Guo S, Ouyang S, Zhang Y, Xu J: Involvement of cell adhesion molecules in polydatin protection of brain tissues from ischemia-reperfusion injury. Brain Res 2006, 1110(1):193-200.

25. Chen L, Han Y, Yang F, Zhang T: High-speed counter-current chromatography separation and purification of resveratrol and piceid from Polygonum cuspidatum. J Chromatogr A 2001, 907(1-2):343-346.

26. Cheng CY, Ho TY, Lee EJ, Su SY, Tang NY, Hsieh CL: Ferulic acid reduces cerebral infarct through its antioxidative and anti-inflammatory effects following transient focal cerebral ischemia in rats. Am J Chin Med 2008, 36(6):1105-1119.

27. Kadhim HJ, Duchateau J, Sebire G: Cytokines and brain injury: invited review. J Intensive Care Med 2008, 23(4):236-249.

28. Tang YH, Zhang SP, Liang Y, Deng CQ: Effects of Panax notoginseng saponins on mRNA expressions of interleukin-1 beta, its correlative factors and cysteinyl-aspartate specific protease after cerebral ischemiareperfusion in rats. Zhong Xi Yi Jie He Xue Bao 2007, 5(3):328-332.

29. Zhang G, Yu Z, Zhao H: Protective effect of paeonol on repeated cerebral ischemia in rats. Zhong Yao Cai 1997, 20(12):626-628.

30. Chan SJ, Wong WS, Wong PT, Bian JS: Neuroprotective effects of andrographolide in a rat model of permanent cerebral ischaemia. $\mathrm{Br} J$ Pharmacol 2010, 161(3):668-679.

31. Yan B, Wang DY, Xing DM, Ding Y, Wang RF, Lei F, Du LJ: The antidepressant effect of ethanol extract of radix puerariae in mice exposed to cerebral ischemia reperfusion. Pharmacol Biochem Behav 2004 78(2):319-325.

32. Chang Y, Hsieh CY, Peng ZA, Yen TL, Hsiao G, Chou DS, Chen CM, Sheu JR: Neuroprotective mechanisms of puerarin in middle cerebral artery occlusion-induced brain infarction in rats. J Biomed Sci 2009, 16:9.

33. Kim YO, Leem K, Park J, Lee P, Ahn DK, Lee BC, Park HK, Suk K, Kim SY, Kim H: Cytoprotective effect of Scutellaria baicalensis in CA1 hippocampal neurons of rats after global cerebral ischemia. $J$ Ethnopharmacol 2001, 77(2-3):183-188.

34. Lee H, Kim YO, Kim H, Kim SY, Noh HS, Kang SS, Cho GJ, Choi WS, Suk K: Flavonoid wogonin from medicinal herb is neuroprotective by inhibiting inflammatory activation of microglia. FASEB J 2003, 17(13):1943-1944.

35. Chan $\mathrm{PH}$ : Reactive oxygen radicals in signaling and damage in the ischemic brain. J Cereb Blood Flow Metab 2001, 21(1):2-14.

36. Wang $Q$, Tang $X N$, Yenari MA: The inflammatory response in stroke. J Neuroimmunol 2007, 184(1-2):53-68

37. Kelly MA, Shuaib A, Todd KG: Matrix metalloproteinase activation and blood-brain barrier breakdown following thrombolysis. Exp Neurol 2006, 200(1):38-49.
38. Cheng CY, Su SY, Tang NY, Ho TY, Chiang SY, Hsieh CL: Ferulic acid provides neuroprotection against oxidative stress-related apoptosis after cerebral ischemia/reperfusion injury by inhibiting ICAM-1 mRNA expression in rats. Brain Res 2008, 1209:136-150.

39. Kao TK, Ou YC, Kuo JS, Chen WY, Liao SL, Wu CW, Chen CJ, Ling NN, Zhang $\mathrm{YH}$, Peng $\mathrm{WH}$ : Neuroprotection by tetramethylpyrazine against ischemic brain injury in rats. Neurochem Int 2006, 48(3):166-176.

40. Hsiao G, Chen YC, Lin JH, Lin KH, Chou DS, Lin CH, Sheu JR: Inhibitory mechanisms of tetramethylpyrazine in middle cerebral artery occlusion (MCAO)-induced focal cerebral ischemia in rats. Planta Med 2006, 72(5):411-417.

41. Lao CJ, Lin JG, Kuo JS, Chiang SY, Chen SC, Liao ET, Hsieh CL: Effect of salvia miltiorrhiza bunge on cerebral infarct in ischemia-reperfusion injured rats. Am J Chin Med 2003, 31(2):191-200.

42. Sze FK, Yeung FF, Wong E, Lau J: Does Danshen improve disability after acute ischaemic stroke? Acta Neurol Scand 2005, 111(2):118-125.

43. Kuang $P$, Tao Y, Shi J: Effect of radix Salviae miltiorrhizae on extracellular adenosine and evaluation of its protective efficacy in ischemic reperfusion rat-microdialysis, HPLC and histopathologic studies. J Tradit Chin Med 1997, 17(2):140-147.

44. Wu W, Kuang P, Li Z: Effect of radix Salviae miltiorrhizae on the gene expression of nitric oxide synthase in ischemic rat brains. J Tradit Chin Med 1998, 18(2):128-133.

45. Xia WJ, Yang M, Fok TF, Li K, Chan WY, Ng PC, Ng HK, Chik KW, Wang CC, Gu GJS, Woo KS, Fung KP: Partial neuroprotective effect of pretreatment with tanshinone IIA on neonatal hypoxia-ischemia brain damage. Pediatr Res 2005, 58(4):784-790.

46. Yu SJ, Kim JR, Lee CK, Han JE, Lee JH, Kim HS, Hong JH, Kang SG: Gastrodia elata blume and an active component, p-hydroxybenzyl alcohol reduce focal ischemic brain injury through antioxidant related gene expressions. Biol Pharm Bull 2005, 28(6):1016-1020.

47. Kim HJ, Lee SR, Moon KD: Ether fraction of methanol extracts of Gastrodia elata, medicinal herb protects against neuronal cell damage after transient global ischemia in gerbils. Phytother Res 2003, 17(8):909-912.

48. Kim HJ, Hwang IK, Won MH: Vanillin, 4-hydroxybenzyl aldehyde and 4hydroxybenzyl alcohol prevent hippocampal CA1 cell death following global ischemia. Brain Res 2007, 1181:130-141.

49. Yu SS, Zhao J, Zheng WP, Zhao Y: Neuroprotective effect of 4hydroxybenzyl alcohol against transient focal cerebral ischemia via antiapoptosis in rats. Brain Res 2010, 1308:167-175.

50. Zheng CJ, Liao WJ, Fan M, Yang WT, Meng LQ: Effects of Angelica sinesis treatment on the expression of flt-1 and flk-1 mRNA after the iscemic brain injury in rats. Zhongguo Ying Yong Sheng Li Xue Za Zhi 2006, 22(4):385-389.

51. Lin Z, Zhu D, Yan Y, Yu B, Wang Q, Shen P, Ruan K: An antioxidant phytotherapy to rescue neuronal oxidative stress. Evid Based Complement Alternat Med 2008

52. Liao SL, Kao TK, Chen WY, Lin YS, Chen SY, Raung SL, Wu CW, Lu HC, Chen CJ: Tetramethylpyrazine reduces ischemic brain injury in rats. Neurosci Lett 2004, 372(1-2):40-45.

53. Oh JK, Hyun SY, Oh HR, Jung JW, Park C, Lee SY, Park JH, Kim SY, Kim KH, Kim YK, Ryu JH: Effects of Anemarrhena asphodeloides on focal ischemic brain injury induced by middle cerebral artery occlusion in rats. Biol Pharm Bull 2007, 30(1):38-43.

54. Shang YZ, Miao H, Cheng JJ, Qi JM: Effects of amelioration of total flavonoids from stems and leaves of Scutellaria baicalensis Georgi on cognitive deficits, neuronal damage and free radicals disorder induced by cerebral ischemia in rats. Biol Pharm Bull 2006, 29(4):805-810.

55. Lee JH, Jung KM, Bae IH, Cho S, Seo DB, Lee SJ, Park YH, Lim KM: Antiinflammatory and barrier protecting effect of Lithospermum erythrorhizon extracts in chronic oxazolone-induced murine atopic dermatitis. J Dermatol Sci 2009, 56(1):64-66.

56. Papageorgiou VP, Assimopoulou AN, Ballis AC: Alkannins and shikonins: a new class of wound healing agents. Curr Med Chem 2008, 15(30):3248-3267

57. Wang Z, Liu T, Gan L, Wang T, Yuan X, Zhang B, Chen H, Zheng Q: Shikonin protects mouse brain against cerebral ischemia/reperfusion injury through its antioxidant activity. Eur J Pharmacol 2010, 643(23):211-217. 
58. Wang C, Zhang D, Ma H, Liu J: Neuroprotective effects of emodin-8-Obeta-D-glucoside in vivo and in vitro. Eur J Pharmacol 2007, 577(13):58-63.

59. Liu QX, Wang B, Liang H, Zhao YY, Liu MJ: Structure identification of jujuboside D. Yao Xue Xue Bao 2004, 39(8):601-604.

60. Guo S, Duan JA, Tang YP, Yang NY, Qian DW, Su SL, Shang EX: Characterization of triterpenic acids in fruits of ziziphus species by HPLC-ELSD-MS. J Agric Food Chem 2010, 58(10):6285-6289.

61. Jiang JG, Huang XJ, Chen J: Separation and purification of saponins from Semen Ziziphus jujuba and their sedative and hypnotic effects. $J$ Pharm Pharmacol 2007, 59(8):1175-1180.

62. Lee JK, Kwak HJ, Piao MS, Jang JW, Kim SH, Kim HS: Quercetin reduces the elevated matrix metalloproteinases-9 level and improves functional outcome after cerebral focal ischemia in rats. Acta Neurochir (Wien) 2011, 153(6):1321-1329.

63. Li H, Deng CQ, Chen BY, Zhang SP, Liang Y, Luo XG: Total saponins of Panax notoginseng modulate the expression of caspases and attenuate apoptosis in rats following focal cerebral ischemia-reperfusion. $J$ Ethnopharmacol 2009, 121(3):412-418.

64. Huang $X$, Tan H, Chen B, Deng C: Influence of astragalosides and Panax notoginseng saponins compatibility on MMP-9 and TIMP-1 after cerebral ischemia-reperfusion in mice. Zhongguo Zhong Yao Za Zhi 2010, 35(16):2187-2191.

65. Chen W, Dang Y, Zhu C: Simultaneous determination of three major bioactive saponins of Panax notoginseng using liquid chromatographytandem mass spectrometry and a pharmacokinetic study. Chin Med 2010, 5:12.

66. Mines M, Ding Y, Fan GH: The many roles of chemokine receptors in neurodegenerative disorders: emerging new therapeutical strategies. Curr Med Chem 2007, 14(23):2456-2470.

67. Adibhatla RM, Hatcher JF, Larsen EC, Chen X, Sun D, Tsao FH: CDP-choline significantly restores phosphatidylcholine levels by differentially affecting phospholipase A2 and CTP: phosphocholine cytidylyltransferase after stroke. J Biol Chem 2006, 281(10):6718-6725.

68. Schwab JM, Beschorner R, Meyermann R, Gozalan F, Schluesener HJ: Persistent accumulation of cyclooxygenase-1-expressing microglial cells and macrophages and transient upregulation by endothelium in human brain injury. J Neurosurg 2002, 96(5):892-899.

69. Dore S, Otsuka T, Mito T, Sugo N, Hand T, Wu L, Hurn PD, Traystman RJ, Andreasson K: Neuronal overexpression of cyclooxygenase-2 increases cerebral infarction. Ann Neurol 2003, 54(2):155-162.

70. Manabe Y, Anrather J, Kawano T, Niwa K, Zhou P, Ross ME, ladecola C: Prostanoids, not reactive oxygen species, mediate COX-2-dependent neurotoxicity. Ann Neurol 2004, 55(5):668-675.

71. Rao AM, Hatcher JF, Kindy MS, Dempsey RJ: Arachidonic acid and leukotriene C4: role in transient cerebral ischemia of gerbils. Neurochem Res 1999, 24(10):1225-1232.

72. Sato S, Takeo J, Aoyama C, Kawahara H: Na+-glucose cotransporter (SGLT) inhibitory flavonoids from the roots of Sophora flavescens. Bioorg Med Chem 2007, 15(10):3445-3449.

73. Cui L, Zhang X, Yang R, Wang L, Liu L, Li M, Du W: Neuroprotection and underlying mechanisms of oxymatrine in cerebral ischemia of rats. Neurol Res 2011, 33(3):319-324(6).

74. Park SJ, Nam KW, Lee HJ, Cho EY, Koo U, Mar W: Neuroprotective effects of an alkaloid-free ethyl acetate extract from the root of Sophora flavescens Ait. against focal cerebral ischemia in rats. Phytomedicine 2009, 16(11):1042-1051.

75. Schoonbroodt S, Ferreira V, Best-Belpomme M, Boelaert JR, Legrand-Poels S, Korner M, Piette J: Crucial role of the amino-terminal tyrosine residue 42 and the carboxyl-terminal PEST domain of I kappa B alpha in NF-kappa B activation by an oxidative stress. J Immunol 2000, 164(8):4292-4300.

76. Kyriakis JM, Avruch J: Mammalian mitogen-activated protein kinase signal transduction pathways activated by stress and inflammation. Physiol Rev 2001, 81(2):807-869.

77. Gao Q, Li Y, Chopp M: Bone marrow stromal cells increase astrocyte survival via upregulation of phosphoinositide 3-kinase/threonine protein kinase and mitogen-activated protein kinase kinase/extracellular signalregulated kinase pathways and stimulate astrocyte trophic factor gene expression after anaerobic insult. Neuroscience 2005, 136(1):123-134.

78. Lu XC, Williams AJ, Yao C, Berti R, Hartings JA, Whipple R, Vahey MT, Polavarapu RG, Woller KL, Tortella FC, Dave JR: Microarray analysis of acute and delayed gene expression profile in rats after focal ischemic brain injury and reperfusion. J Neurosci Res 2004, 77(6):843-857.

79. Simi A, Ingelman-Sundberg $M$, Tindberg N: Neuroprotective agent chlomethiazole attenuates c-fos, c-jun, and AP-1 activation through inhibition of p38 MAP kinase. J Cereb Blood Flow Metab 2000, 20(7):1077-1088.

80. Piao HZ, Jin SA, Chun HS, Lee JC, Kim WK: Neuroprotective effect of wogonin: potential roles of inflammatory cytokines. Arch Pharm Res 2004, 27(9):930-936.

81. Liu Y, Zhang XJ, Yang CH, Fan HG: Oxymatrine protects rat brains against permanent focal ischemia and downregulates NF-kappaB expression. Brain Res 2009, 1268:174-180.

82. Liu J, Jin DZ, Xiao L, Zhu XZ: Paeoniflorin attenuates chronic cerebral hypoperfusion-induced learning dysfunction and brain damage in rats. Brain Res 2006, 1089(1):162-170.

83. Cho J, Lee HK: Wogonin inhibits ischemic brain injury in a rat model of permanent middle cerebral artery occlusion. Biol Pharm Bull 2004, 27(10):1561-1564.

84. Chen C, Venketasubramanian N, Gan RN, Lambert C, Picard D, Chan BPL, Chan E, Bousser MG, Xuemin S: Danqi Piantang Jiaonang (DJ), a traditional Chinese medicine, in poststroke recovery. Stroke 2009, 40(3):859-863.

85. Young SHY, Zhao Y, Koh A, Singh R, Chan BPL, Chang HM Venketasubramanian N, Chen C: Safety profile of MLC601 (Neuroaid) in acute ischemic stroke patients: A Singaporean substudy of the Chinese medicine neuroaid efficacy on stroke recovery study. Cerebrovasc Dis 2010, 30(1):1-6.

86. Venketasubramanian N, Chen CLH, Gan RN, Chan BPL, Chang HM, Tan SB, Picard D, Navarro JC, Baroque II AC, Poungvarin N, Donnan GA, Bousser MG: A double-blind, placebo-controlled, randomized, multicenter study to investigate CHInese Medicine Neuroaid Efficacy on Stroke recovery (CHIMES Study). Int J Stroke 2009, 4(1):54-60.

87. He L, Chen X, Zhou M, Zhang D, Yang J, Yang M, Zhou D: Radix/Rhizoma Notoginseng extract (Sanchitongtshu) for ischemic stroke: A randomized controlled study. Phytomedicine 2011, 18(6):437-442.

doi:10.1186/1749-8546-6-26

Cite this article as: Su and Hsieh: Anti-inflammatory effects of Chinese medicinal herbs on cerebral ischemia. Chinese Medicine 2011 6:26.

\section{Submit your next manuscript to BioMed Central and take full advantage of:}

- Convenient online submission

- Thorough peer review

- No space constraints or color figure charges

- Immediate publication on acceptance

- Inclusion in PubMed, CAS, Scopus and Google Scholar

- Research which is freely available for redistribution

Submit your manuscript at www.biomedcentral.com/submit
C Biomed Central 